\title{
Making Scientific and Technical Materials Pervasively Accessible
}

\author{
Jason J.G. White* \\ Educational Testing Service, Princeton, NJ, USA
}

\begin{abstract}
In this paper, the question is explored of what policies, standards and practices are desirable to ensure that hardware, software and publications in the sciences and associated disciplines are created from the outset to be accessible to people with disabilities. Insight into this question can be obtained by considering the unique accessibility challenges that these materials pose, including complexities of notation, language, and graphical representation.

Having analyzed what sets this problem apart from broader issues of accessibility, the advantages and limitations of current international standards are reviewed, and contemporary developments in standards and policies are considered from a strategic perspective. These developments include the establishment of accessibility requirements for e-books and e-readers under the European Accessibility Act, the potential role of process-oriented accessibility standards such as ISO/IEC 300711:2019, and opportunities for enhancing the standards applicable to scientific materials via future revisions of the World Wide Web Consortium's Web Content Accessibility Guidelines (WCAG). The accessibility of scientific and technical content is ultimately supported by several interrelated human rights recognized in international disability rights law, which constitute a foundation for further evaluation and development of policies. It is argued that attaining pervasive accessibility in scientific and technical fields requires an unprecedented level of commitment and collaboration among educators, scientists, content and software producers, regulators, and people with disabilities.
\end{abstract}

*Corresponding Author, Jason J.G. White (jjwhite@ets.org)

Submitted February 11, 2021

Accepted April 25, 2021

Published online July 21, 2021

DOI: $10.14448 /$ jsesd.13.0006 


\section{Introduction to the Problem}

For any student or professional who is working in a science, technology, engineering or mathematics (STEM) field, access to discipline-related resources is crucially important. This need first arises at the primary school level, for example in elementary arithmetic, and evolves as a student progresses through secondary school, university study, and onward to a career. Frequently, these resources comprise scientific or technical publications, such as books and articles, whether written to serve educational purposes, as in textbooks, or to report the findings of research. Documents of a more informal or ephemeral nature, for example exercise sets, notes, and presentation slides, often occur in educational environments and in the workplace. Due to the development and adoption of information and communication technologies in recent decades, software occupies an ever larger role in STEM-related learning and practice. Among the illustrations that could be given, one may consider the educational use of scientific calculators and symbolic algebra systems, the operation of software that controls laboratory equipment and reports data, and the application of word processors or typesetting tools supporting mathematical notation to document creation. In educational research and practice, interactive applications are growing in both sophistication and prominence, as exemplified by the use of interactive science simulations, ${ }^{1}$ or by the development of intelligent tutoring systems (Hansen, Zapata-Rivera, \& White, 2018) in scientific and technical fields.

Whereas conventionally published materials were once destined primarily for printing, they are now produced and increasingly, delivered electronically. Multimedia presentations, particularly video, have become ubiquitously available and easier to produce. A growing body of scientific and technical knowledge is available via the World Wide Web. The tools offered for scientific communication have also expanded to include, for example, audio and video-based teleconference facilities in which communication and collaboration on shared documents can take place synchronously. In this environment, access to an increasing variety of software and digital content is a practical necessity in education and employment alike. This is no less true of those who have disabilities than it is of students and professionals in STEM disciplines generally. In addition, digital products and services can be more accessible to people with disabilities than their traditional counterparts, thus offering opportunities to achieve equality and inclusion to an unprecedented extent.

Whereas traditional alternative formats - manually transcribed braille, large print, and audio-principally serve the needs of users who are blind or who have low vision, and to a lesser extent those with other print disabilities, digital materials can be much more versatile. Multimedia content and

\footnotetext{
${ }^{1}$ An approach to enhancing the accessibility of such simulations is described in Smith, Greenberg, Reid, and Moore (2018).
}

interactive user interfaces provide opportunities to present information and to support the performance of tasks in ways that are customized to the needs of the individual to an extent which conventional formats cannot equal. The instant conversion of digital materials via assistive technologies for presentation to the user with a disability provides immediacy of access that is unattainable with the slow and laborious processes of conventional alternative format production. Whether such superior access by digital means is achieved in specific circumstances depends substantially on the knowledge and skills of the person with a disability in the use of technology, which in turn can vary according to socioeconomic conditions and the availability of appropriate resources or learning opportunities. Of decisive importance however are the accessibility-related features of the products and services themselves - the extent to which they are designed and constructed to address needs arising from physical, sensory, cognitive or other forms of disability. ${ }^{2}$ These considerations, and the laws and policies surrounding them, are the subject of this paper, leaving aside the role of educational institutions, support services, and socioeconomic inequalities in shaping the motivations, aptitudes, and technological skills that the user brings to the task. It should further be recognized that features designed to enable access by people with disabilities can enhance the usability of a product or service by people who would not be regarded as having a disability. For example, captions can facilitate understanding of multimedia content by anyone placed in a noisy environment. It is also important to acknowledge that the needs arising directly or indirectly from disability are combined with other aspects of a person's background, such as linguistic competence in the language in which materials are delivered, to influence what he or she can achieve in practice with digital content.

Thus, although addressing problems of accessibility would contribute to overcoming barriers encountered by people with disabilities in education and employment, it should be regarded as a part of a larger project of social and, importantly in the present context, educational justice. Providing accessible educational materials would not, for instance, necessarily address the expectancy beliefs that Schneiderwind and Johnson (2020) hypothesize may dissuade some students with disabilities from pursuing further study in STEM disciplines. Nor would it overcome differences in educational achievement associated with socioeconomic inequal-

\footnotetext{
${ }^{2}$ This general characterization of the problem of accessibility sufficiently identifies the subject-matter for the purposes of the current discussion. There exist a variety of definitions of "accessibility", "universal design", "inclusive design" and other associated terms, which have similarities as well as important differences. A critical analysis of these definitions lies beyond the scope of this paper. An overview of them in historical context can be found in Persson, Åhman, Yngling, and Gulliksen, 2015.
} 
ities, ${ }^{3}$ which not only affect students with disabilities in similar respects to the student population generally, but which can also have disability-related manifestations. In a system which privileges individual advocacy, for instance, the unequal capacities of differently resourced families to advocate for meeting the educational needs of a child with a disability may significantly influence outcomes. ${ }^{4}$ Although accessibility is typically understood as a matter of discriminatory conduct, for example by an employer or an educational institution, the related issues alluded to here introduce normative questions, beyond the limits of this paper, about what constitutes educational justice, particularly for students with disabilities. ${ }^{5}$ Broader issues of educational and socioeconomic inequality are important; but, as has been indicated, they are also distinct problems, with their own implications for policy, from the more narrowly focused questions of accessibility considered here. Disability-related accessibility issues are uniquely grounded in the human rights of people with disabilities, in requirements of non-discrimination, and in international technical standards which have influenced policy development. They are thus distinct concerns, even though, as actually experienced in practice, they may be inseparable from other factors such as socioeconomic conditions or the availability of educational opportunities, that also raise issues of policy and social justice. ${ }^{6}$ Although it is difficult to establish a clear demarcation between accessibility and associated concerns of general usability or the effects of non-disability-related conditions, obliterating this distinction entirely risks marginalizing some of the particular questions of inclusion and civil rights that are specific to disability as a concept and as it is experienced (Ellcessor, 2015).

For the full potential of information and communications technology in equalizing access to be achieved, therefore, scientific and technical materials, including a wide variety of hardware, software and documents, need to be accessible to people with disabilities. Attaining adequate accessibility demands a commitment of resources and expertise that are best applied throughout the conception, design, development and maintenance of a product or service. The alterna-

\footnotetext{
${ }^{3}$ For an informative introduction to these inequalities from the standpoint of educational justice, see Brighouse, Ladd, Loeb, and Swift (2018, chapter 3).

${ }^{4}$ For an empirical investigation of this phenomenon in the context of U.S. educational policy, see Ong-Dean (2009).

${ }^{5} \mathrm{~A}$ range of normative positions concerning the demands of educational justice is elaborated rigorously in the contributions to the contemporary debate collected in Meyer (2014) The challenge posed to such accounts by students with disabilities - in particular, those with cognitive disabilities - is illuminated in Ahlberg (2014) and Taylor (2018).

${ }^{6}$ It has further been argued (Liasidou, 2013) that addressing intersectional issues is necessary to achieving justice for students with disabilities, and hence the over-all problem is substantially more complex than the examples presented here may suggest.
}

tive of modifying an existing product to satisfy accessibility requirements generally involves changing decisions related to design and construction that have already been taken, and therefore increases costs by comparison with building it to be accessible from the outset. ${ }^{7}$ In some cases, the additional costs may be high, for example if nothing short of a fundamental redesign can satisfy accessibility requirements, or if a software system has been built from components that do not support assistive technologies, and thus the only remedy is to reimplement the user interface entirely. Furthermore, to contain the costs of rectifying an existing product, decisions may be made that, while technically satisfying accessibility requirements, result in a user interface which imposes unnecessary burdens and inconveniences upon the user, for example by unduly complicating interaction and navigation in the performance of common tasks.

From the standpoint of policy, these reasons justify ensuring that digital materials are, so far as the state of current knowledge permits, created from the outset to be broadly accessible to people with disabilities. As is discussed later in this paper, considerations of inclusion and nondiscrimination establish a strong rationale, reflected in norms of international human rights, for applying such a policy to a wide variety of STEM-related products and services that may be encountered in such contexts as education and employment. To evoke the terminology promoted by Benetech (Benetech, 2015; Wentz et al., 2011), the objective is thus to ensure that digital products and services are "born accessible" to users with disabilities. Since this argument is applicable to information and communication technologies in general, it clearly holds in connection with the narrower class of materials specialized for STEM disciplines.

Cross-disability accessibility ought therefore to become a normal and expected characteristic of information and communication technology-based products and services that is included appropriately at all stages of their development as a matter of routine. It should not be an exceptional feature, nor added inadequately and belatedly. To achieve this desirable outcome, which shall for purposes of this paper be referred to as a state of pervasive accessibility, those responsible for creating or maintaining digital products and services require the resources, the skill, and the motivation to address the needs of a broad diversity of potential users, including people with disabilities. Various means are available for ensuring that these conditions are put in place. Policies favoring inclusion and nondiscrimination, whether established by governmental regulation or adopted by organizations voluntarily, clearly have a major role. Education of individuals responsible for planning, allocating resources to and undertaking the development of hardware, software and documents in aspects of accessibility relevant to their

\footnotetext{
${ }^{7}$ For a critical response to the phenomenon of retrofitted accessibility in relation to anti-discrimination law in the United States, see Wentz, Jaeger, and Lazar (2011).
} 
work is clearly a necessity. ${ }^{8}$ Tools and materials, including content authoring software ${ }^{9}$ and software components such as libraries offered for use in building applications, ${ }^{10}$ can also facilitate the creation of accessible products.

International technical standards related to the accessibility of the Web and of application software can be understood as serving three central functions. ${ }^{11}$ First, they set forth norms that may be incorporated into policies and practices. Second, they document solutions to significant problems of accessibility, and, by so doing, equip practitioners with ready-made approaches that can be applied to the construction of products which are built to be cross-disability accessible. Third, standards help to ensure the compatibility of the accessibility-related features of products and components developed by different organizations. For instance, the accessibility of a Web page to the user of an assistive technology may depend on common standards implemented by the authoring tool with which the page is created, third-party library components used in its construction, the browser in which it is rendered on behalf of the user, and the support provided by the assistive technology itself.

Beyond the education of participants in the hardware and software development process, and the refinement of technical standards, it is clearly important to invest in the further development of assistive technologies. For example, notwithstanding the various approaches that have been documented, and patented, in recent decades, tactile displays capable of presenting braille and graphical content have not become available to the public at an affordable cost. The potential of machine learning-based artificial intelligence to simplify and facilitate interaction by people with a wide variety of learning or cognitive disabilities similarly remains to be developed. Indeed, machine learning offers untapped opportunities to enhance accessibility both in the process of digital content creation, and as an assistive technology operated directly by users. Promotion of practices supportive of accessibility is also a necessary element of an effective strategy.

As will be further explained in the following discussion, digital artifacts such as documents and software designed to be used in connection with STEM domains often have distinctive characteristics that yield specific accessibility requirements. For example, in a software system such as a word processor or an educational application, it is typically neces-

${ }^{8}$ For a thoughtful discussion of what is needed to build a "pedagogical culture" of education in accessibility, see Lewthwaite and Sloan (2016).

${ }^{9}$ Aspects of the accessibility of such software are standardized in World Wide Web Consortium, 2015.

${ }^{10}$ There is currently no international standard for the accessibility of these components. However, some aspects of support for assistive technology are addressed for Web-based applications in World Wide Web Consortium, 2017.

${ }^{11}$ Many of the more prominent standards are cited in this paper. sary to support the presentation of mathematical notation to the user, and to provide an interface through which it can be edited and manipulated - a requirement that does not apply to general-purpose technologies that are not intended to accommodate STEM disciplines. Consequently, the tools and strategies available to designers and implementers of accessible digital artifacts for satisfying users" accessibility needs can only be successfully applied to the STEM-specific aspects of these products if they offer solutions to the issues that arise in relation to scientific and technical disciplines. Correspondingly, the effectiveness of policies in ensuring that such products satisfy the access needs of students and professionals with disabilities who work in STEM fields, depends on whether the STEM related requirements are properly addressed. For example, an adequate policy would explicitly or implicitly mandate that the features of the aforementioned word processor or educational application which are concerned with the presentation and editing of mathematical notation be designed to support effective use by people with a variety of disabilities, including users who are blind, who have low vision, or who have dyslexia.

Thus, a fundamental question concerning the accessibility of digital artifacts related to STEM disciplines is what provision should be made in applicable standards and policies to address the particular challenges that emerge in these domains. Although it is apparent that standards, policies, and education of practitioners are all crucial ingredients of sustainable and effective solutions to problems of accessibility, it is also necessary for these factors to be evaluated and improved from a STEM-specific perspective. Policies and practices, moreover, should be informed by research. There is a growing body of research in accessibility, including human-computer interaction, that can inform the development of accessible materials in STEM disciplines. Much of this work is devoted to narrowly defined, concrete problems. This observation raises the question of the extent to which research relevant to accessibility can be synthesized to enable the development of technically well founded and evidence-based solutions to the problems that need to be solved in order for accessible, STEM-related materials to be designed and implemented on a widespread basis as a matter of routine. Seldom are research findings in information and communication technology accessibility synthesized to create guidance for practitioners, and it is unclear what gaps exist in the established body of research that ought to be filled in service of the need for better policies and practices. In determining priorities for future research, it would be prudent to consider them in the context of the larger enterprise of building accessibility into the design and development of digital materials, including those addressed to students and professionals in scientific and technical domains. It is also evident that the totality of the accessibility problem, and the strategies needed to address it as a unified whole, are rarely considered explicitly in the literature, either in general or in the context of STEM fields. The present contribution 
takes a first step toward redressing this imbalance of emphasis by commenting on some relevant standards, policies, and norms of international disability rights law from the standpoint of institutionalizing the production of accessible STEM materials. The accessibility-related characteristics of STEM materials are first identified analytically by characterizing their distinctive communicative features. Relevant standards and policies are then discussed, supporting provisional conclusions about the nature of the task that lies ahead in achieving pervasive accessibility.

\section{Accessibility-Relevant Properties of STEM Ma- terials}

Scientific and technical fields have distinct linguistic usages, notations, and conventions of graphical representation. The capacity to read, understand, and produce the appropriate forms of expression is essential to working effectively in such a discipline. Consequently, these representations of scientific and technical ideas are reflected in documents, in multimedia presentations such as videos, and in the functionality provided by software. They also lead to technical challenges of access for people with disabilities. By considering these aspects of STEM content, which together distinguish it from more general, non-technical materials, it is possible to confine the scope of the discussion to the issues of accessibility that are specific to STEM disciplines. Of course, in actual content, these STEM-related issues are intertwined with considerations that apply to the accessibility of digital materials in general. However, for the analytical purpose of examining standards and policies from the standpoint of STEM disciplines, it is useful to concentrate on the challenges specific to the distinctive characteristics of materials written in these fields.

According to Mason and Hedin (2011), scientific writing has features that distinguish it from simpler narrative or descriptive text, and which pose difficulties for people with learning disabilities. ${ }^{12}$ These characteristics include structural variability, conceptual density (the rate at which new terms and concepts are introduced, combined with increasing dependence on abstract logical and causal relations), reliance on technical vocabulary, and invocation of the reader's ability to draw inferences based on assumed, prior knowledge. The use of technical vocabulary can also introduce difficulties for people whose disabilities are unrelated to learning or cognition, for example by placing demands on the accuracy of text to speech technology or speech recognition systems.

Assistive technologies have a limited but significant role in addressing these challenges. The text, including technical terminology, may be accessed via text to speech software as part of a screen reader, or of a read-aloud system offering text to speech with synchronized highlighting. Correct

\footnotetext{
${ }^{12}$ They are also typical of academic writing more generally. See Silliman and Wilkinson (2015) for a discussion.
}

pronunciation is desirable, in that it facilitates understanding of what is read; however, general-purpose software may not achieve an appropriate level of accuracy without provision of a supplemental pronunciation lexicon ${ }^{13}$ or without tailoring the software for use in STEM domains. Similarly, automatic speech recognition can be used as an assistive technology - specifically, as a means of input by people with disabilities - or as a tool for content creators to use in automatically generating captions. Questions of accuracy emerge, however, in the recognition of scientific and technical vocabulary. If automatic translation of text into sign languages becomes sufficiently reliable to be useful in academic and professional contexts, ${ }^{14}$ it is easily foreseeable that the vocabulary of STEM disciplines will need to be taken into account to achieve adequate translations.

The difficulties of conceptual density, text structure, and dependence on antecedent knowledge are more amenable to solutions that modify or supplement the text, rather than to the use of assistive technology. Although text simplification and summary generation methods are an active field of research in natural language processing, and may perhaps be useful in practice, considerable research and technical development would be needed to achieve the level of accuracy required for general use in educational or professional settings by people with learning or cognitive disabilities. Glossaries defining technical terms can be provided, either manually or, in principle at least, through the use of appropriate dictionaries. However, much of the responsibility of communicating clearly and simply evidently rests with the authors of scientific and technical texts. These qualities are also challenging to define and to verify, which in turn strengthens a reluctance by standards organizations and governments to include clarity and simplicity requirements in accessibilityrelated standards or policies. Rewriting of existing texts could be avoided by providing supplemental explanations and illustrations, but the efficacy of these solutions in enhancing comprehension - especially by people with learning disabilities - would need to be empirically established in a variety of educational and non-educational contexts if they were to be recommended as general accessibility requirements.

Specialized notations are used extensively in mathematics and the sciences. Mathematical notation and chemical notation are obvious cases. Phonetic transcription in linguistics, and programming language source code in computer science could also be included within this category. Adequate access to notation by a person with a disability necessitates the ability to read, understand, manipulate, and write it effectively. The accessibility-related issues associated with

\footnotetext{
${ }^{13}$ The Pronunciation Lexicon Specification (PLS) provides a standardized mechanism for doing so (World Wide Web Consortium, 2008a).

${ }^{14} 14 \mathrm{~A}$ recent overview of the technical challenges associated with automated sign language translation and production is provided in Bragg et al. (2019).
} 
mathematical notation are relatively well understood, and have been treated extensively in the literature (Soiffer \& Noble, 2019; White, 2020). Conversion of the notation into a format that the user can perceive and understand is of crucial significance. Visual enlargement, spoken presentation, and automatic braille transcription are the most common strategies employed to satisfy this need. Reading and navigation of mathematical expressions according to their structural components is typically also supported by screen readers. For people who have dyslexia, synchronized highlighting of the notation, combined with text to speech, may be an effective solution (Lewis, Noble, \& Soiffer, 2010). Support for writing, modifying, and manipulating the notation is generally provided in software such as mathematical notation editors that can be integrated into applications. The accessibility of these tasks, including their implications for the cognitive demands to which the user is subject, has also been addressed in the literature (e.g., Fajardo Flores \& Archambault, 2012; Stöger, Batusic, Miesenberger, \& Haindl, 2006; Stöger, Miesenberger, \& Batušić, 2004).

Notations specific to other disciplines, such as chemistry, lead to similar challenges. Phonetic transcription and computer program source code are less complex cases, but they nevertheless require special treatment, for example in generating correct spoken presentation, including appropriate pronunciation. A further example of an issue of accessibility which is specific to a given notation arises in the case of programming languages in which indentation is semantically significant. Details of the indentation of each line of code need to be conveyed to technologies such as screen readers, which then require the ability to present this information efficiently to the user, for example by announcing the indentation of each line as the user interactively reads and edits the text. Without a reliable indication of the indentation, the block structure of the program cannot be easily discerned non-visually.

Graphical representations of information are commonplace in STEM fields. Issues of color contrast and of retaining adequate image quality upon enlargement are important considerations for users who have low vision. Those who are color blind need information to be conveyed by means other than color alone. More complex challenges derive from the need of users who are blind for nonvisual access to information that is communicated graphically, and for means of creating and modifying graphical content. Tactile graphics, haptics, textual descriptions of images, and sonification are well understood nonvisual alternatives to graphical representation. Dynamic graphical content, such as a science simulation, can be presented by offering descriptions and interactive controls that can be operated via a screen reader. Strategies for designing user interfaces that support the nonvisual creation or modification of graphical content are less well defined, meriting further research. Refreshable tactile graphics displays have the potential to solve this problem in a broad range of cases by allowing the user to draw and ma- nipulate diagrams interactively on the surface of the device, which are immediately represented in the associated software. Basic capabilities have already been demonstrated (Orbit Research, n.d.; Völkel, Weber, \& Baumann, 2008), and further refinement of display hardware and of user interactions is to be expected. ${ }^{15}$

As this discussion has shown, the aspects of STEM materials which create specific challenges for accessibility can arise in a variety of contexts. The tasks to be performed by the user are likewise diverse. In general, linguistic complexity, specialized notations, and graphical representations may appear in purely static forms, as in a book or article, or in multimedia content such as a video. They may also be presented in a real-time communication tool such as a teleconference, or an interactive application such as a simulation. As has been noted, interactive creation and modification of these materials is central to effective scientific or technical communication, and should thus be made generally accessible to users with disabilities. The characteristics typical of STEM materials are not unique to these disciplines. A specialized notation occurs in music, for example. Graphics are used elsewhere, and there are humanities disciplines that exhibit linguistically complex expository writing. Nevertheless, these means of expression, often occurring together, make the task of creating accessible documents and applications in STEM fields unique. Combining STEM-specific aspects of content with standard interactive features can also raise difficulties, such as the problems for screen reader navigation that emerge if mathematical expressions are used as labels of interactive controls, for example radio buttons. The issue of integrating notations and graphics properly with general-purpose features of documents and application software should not be overlooked.

\section{Accessibility Standards and the Distinctive Char- acteristics of STEM Materials}

The practical influence of technical standards over the design and implementation of accessible products and services is substantial. This influence arises in part from the incorporation of such standards into governmental policies, most prominently, requirements in the United States and the European Union with respect to the accessibility of technologies used in the public sector (European Telecommunications Standards Institute, 2018; European Union, 2016; United States, 2017). Further development of technical standards by government, or citation of standards created elsewhere, can reasonably be anticipated. Individuals and organizations have clear motivations to adopt technical standards

\footnotetext{
${ }^{15}$ Although emphasis here is placed on graphical content, the access difficulties associated with non-speech audio should also be acknowledged. For the most part, captions or sign language communication describing the audio can be given. However, some sounds are likely to be difficult to describe, such as certain stimuli used in psychological experiments.
} 


\section{Journal of Sclence Education}

independently of their role in government policy. These grounds include considerations of compatibility with tools and technologies supporting the standards, and the perceived authority that the standards carry in reflecting accessibility - related-expertise. Due to their influential role in practice and policy, understanding the extent to which accessibility standards support materials in STEM domains is a valuable step toward identifying opportunities for improvement. Consideration of this question also enables clarification of what the standards require, together with the elucidation of interpretive ambiguities that may arise.

As the World Wide Web Consortium's Web Content Accessibility Guidelines (WCAG) (World Wide Web Consortium, 2018b) are widely cited in policies and by practitioners of accessibility, they are the suitable focus of a brief review from the standpoint of STEM content. The scope of the analysis undertaken here is confined by the accessibilityrelated properties of STEM materials already considered. Thus, only aspects of WCAG that are specially applicable to the distinctive characteristics of STEM content are reviewed in the following discussion. Version 2.1 of WCAG is current, although version 2.2 is, at the time of writing, nearing completion. The normative requirements of WCAG are expressed as success criteria-assertions that must be true of Web content to which they are applicable in order for it to conform to the Guidelines. The success criteria are divided into three successive levels of conformance, A, AA, and AAA, respectively. These levels build upon one another to achieve progressively more effective access for people with physical, sensory, learning, or cognitive disabilities. Policies have generally converged on requiring conformance to WCAG at level AA. Implementation of level AAA success criteria is of course desirable in improving the accessibility of content, but it is not mandated by public policies that cite the Guidelines. ${ }^{16}$

The success criteria are organized according to more general "guidelines", which are themselves placed under four broad "principles" of accessibility. These principles assert that Web content should be "perceivable", "operable", "understandable", and "robust". Although the principles are important in understanding WCAG, the success criteria are what ultimately determine whether the requirements of the standard have been met.

\section{Linguistic Complexity}

All of the success criteria that have the potential to apply to the distinctive uses of language occurring in scientific and technical contexts arise at level AAA. It follows that addressing this aspect of STEM materials lies beyond the limits of policies that depend on WCAG. Furthermore, interpretation of the relevant level AAA success criteria reveals their

\footnotetext{
${ }^{16}$ Quotations of and references to success criteria in the following analysis are drawn from World Wide Web Consortium (2018b).
}

limited application to improving the comprehensibility of scientific and technical writing.

Success criterion 1.2.6 specifies the provision of sign language interpretation for the audio track of prerecorded video content. This is a level AAA success criterion, whereas captions must be provided at level A (success criterion 1.2.2). Thus, any video content that conforms to WCAG must provide captions, and may optionally offer a sign language interpretation of the audio track as well. There is some evidence that including captions in video content that also provides sign language interpretation can improve comprehension (Debevc, Milošević, \& Kožuh, 2015; Yoon \& Kim, 2011), but this is not the same question as whether sign language should be provided, given that captions must be. Further, it has also been suggested (Marschark et al., 2009) that the difficulties often encountered by students who are deaf in reading text may be attributable more to general issues of linguistic competence applicable to text and sign alike, than to problems of second language acquisition or of reading written material. In so far as this is true, there are some people for whom complementing captions with sign language would remain insufficient to address the challenges in accessing the linguistic components of multimedia content. The level AAA designation of the success criterion further limits the extent of its likely implementation.

Success criterion 3.1.3 requires "a mechanism" to be "available for identifying specific definitions of words or phrases used in an unusual or restricted way, including idioms and jargon". A glossary, for example, would serve as a means of satisfying this requirement. It is important to recognize, however, that the definition of "used in an unusual or restricted way" (World Wide Web Consortium, 2018b, § 6) greatly confines the scope of the success criterion. The example included with the definition clarifies that if the context in which a word or phrase is used is sufficient to indicate which definition should be applied, then no support need be provided for identifying the definition. For example, if the word "field" is used in the context of a discussion of the mathematical properties of the real numbers in an undergraduate mathematics text, then the fact that it refers to the algebraic structure is made clear from the context. Hence, the requirement set forth in the success criterion does not apply, as the use of the term is not "unusual" or "restricted". Indeed, it is typical, conventional usage of terminology in an undergraduate mathematics textbook. Many instances of specialized vocabulary used in STEM disciplines would similarly not need to be associated with disambiguating definitions in order to satisfy the WCAG requirement, as the success criterion is concerned with atypical usage, rather than with the extent to which the terminology used is likely to be understood by members of the author's intended audience. In sum, success criterion 3.1.3 is of relatively little value in furnishing definitions of scientific and technical terms. If such definitions would significantly enhance accessibility, it may be possible to develop software that could 
determine the context and annotate technical terms with links to their corresponding definitions. To what extent this could be accomplished accurately is a suitable matter for research in natural language processing. To what extent it would be useful is a question for investigation regarding the needs of people with learning and cognitive disabilities who are studying or working in STEM fields.

Success criterion 3.1.4 requires a "mechanism" to be available "for identifying the expanded form or meaning of abbreviations". Clearly, only specialized usages that take the form of abbreviations are subject to this requirement; it does not apply generally to specialized scientific or technical vocabulary. As is true of other success criteria, the "mechanism" may be provided within the document or application software, but it may also be supplied by the underlying platform or an assistive technology. The applicability of this success criterion to material in STEM disciplines is likely to be relatively confined.

Success criterion 3.1.5 specifies that text which requires "reading ability more advanced than the lower secondary education level after removal of proper names and titles" be accompanied by a "version that does not require reading ability more advanced than the lower secondary education level". Alternatively, "supplemental content" may be provided that "illustrates" or "clarifies" the material. Although the text of the success criterion is arguably ambiguous in this respect, it is reasonable to interpret it as requiring the supplemental content to necessitate no more than a lower secondary education level of reading ability. Satisfying this success criterion could do much to improve the readability of text in scientific or technical disciplines, particularly if the text written at the lower secondary education level is appropriately crafted to address the problems of conceptual density, technical vocabulary, and assumed prior knowledge that have been identified as barriers to understanding. However, the feasibility of meeting the requirement across a broad range of STEM materials is highly doubtful, not only for practical reasons, but also in principle. The problem, in essence, is that there are many scientific and technical propositions which are inexpressible in text that demands no more than a reading ability at the lower secondary education level.

There is also the risk that providing supplementary clarifications or explanations may do more to confuse a reader with a learning or cognitive disability than it serves to clarify the meaning. Further, satisfying the success criterion, as verified for example by a readability analysis that measures surface features of linguistic complexity such as word and sentence length, does not guarantee that the resulting text is clear, accurate, or likely to be understood by readers with learning disabilities. For these reasons, this success criterion is likely to be applicable only in a narrow range of cases. In the context of STEM disciplines, it would be useful to identify more clearly the circumstances in which this requirement is efficacious in improving accessibility, and the extent to which it can be used to facilitate comprehension, especially in educational environments.

Success criterion 3.1.6 provides for a "mechanism" that identifies "specific pronunciation of words". However, the pronunciation need only be identified if the "meaning of the words, in context, is ambiguous without knowing the pronunciation". Homonyms, at least in some contexts, would qualify. However, it is arguable that words such as scientific and technical terms that are consistently mispronounced by text to speech systems would generally not meet the ambiguity requirement of the success criterion. Thus, it appears that the success criterion does little to improve the pronunciation of specialized vocabulary by general-purpose text to speech systems used in read-aloud tools and screen readers.

Thus, although there are success criteria in WCAG 2.1 that can help to clarify the textual components of scientific and technical materials, they all occur at level AAA, placing them beyond the reach of current policies based on the Guidelines. The scope and efficacy of these success criteria remain unclear, although some benefits can reasonably be hypothesized, particularly in educational settings, and especially to students with learning or cognitive disabilities. As the preceding review shows, each of the success criteria is subject to substantial qualifications and limitations. Significantly, however, measures taken to improve the readability of text can benefit all users, not merely those with disabilities, an advantage that further strengthens the argument for their wide application. Further investigation of what steps can be taken by document authors and in assistive technologies to improve the comprehensibility of text, including scientific and technical content, seems clearly warranted.

\section{Specialized Notation}

Strategies that can be used by applications, Web browsers and assistive technologies to make specialized notations accessible to people with disabilities have already been characterized: use of enlargement, text to speech, braille mathematics codes, structured navigation, and synchronized highlighting. To carry out most of these strategies, it is necessary, or at least highly desirable to have access to a structured, symbolic representation of the specialized notation. This is clearly true of mathematical notation, for which the Mathematical Markup Language (MathML) (World Wide Web Consortium, 2014) serves as the standard symbolic representation, not only on the Web but, increasingly, in electronic documents such as digital publications as well as in application software. The symbolic representation, of course, needs to be available to the software that processes the notation in order to make it accessible to the user, for example, a read-aloud tool or a screen reader. However, this fundamental requirement is not well supported by WCAG, a limitation that leads to problems in practice as well as in principle. The case of mathematical notation as represented on the Web and in digital publications amply illustrates the 
difficulties. Due to inadequacies and inconsistencies in support for MathML by Web browsers, it is customary among certain publishers to deliver mathematical notation only as images, excluding the underlying MathML, and therefore depriving assistive technologies or other software running on the user's behalf of access to the symbolic representation. Alternative text, written in a natural language such as English, may be supplied along with the images. This approach precludes automated translation to mathematical braille by a screen reader, structured navigation, and synchronized highlighting based on the structure of the expression. Unless a vector-based graphics format is used, enlarging the mathematical content may result in a degradation of image quality. It should also be noted that MathML can be visually hidden while remaining accessible to assistive technologies, enabling it to be supplied in a document alongside a graphical representation of the notation. This solution greatly alleviates problems of compatibility with software that lacks sufficient support for MathML. Thus, a strong case can be made for establishing a policy of delivering MathML in scientific and technical content that is implemented using Web technologies, principally HTML.

That WCAG 2.1 does not require the inclusion of structured, symbolic representations of notational content is clear. If the notation is supplied as an image for which there is a text alternative, then success criterion 1.1.1 (a level A requirement) is satisfied. Subject to specified exceptions, success criterion 1.4.5 requires text to be "used to convey information, rather than images of text". However, this requirement only applies if "the technologies being used can achieve the visual presentation". This qualification allows authors of scientific and technical content to decide that MathML is not among "the technologies being used" in delivering their content, and hence that the success criterion is inapplicable. Even if text, rather than an image of text must be used, this still falls short of requiring a structured, symbolic representation of the notation to be supplied, in particular, its MathML form. Success criterion 1.3.1 (level A) states that "[i]nformation, structure, and relationships conveyed through presentation can be programmatically determined or are available in text". While the first option for satisfying this requirement-programmatic determination-is promising, in that it could be understood as demanding a symbolic representation of the notation, the second option - availability in text - can be met by supplying a textual description. Thus, nothing in success criterion 1.3.1 excludes the use of images, with associated descriptions, to convey mathematical or other specialized notations. Indeed, this practice, though objectionable, remains compatible with WCAG at all three levels of conformance. With respect to notations, including mathematics and chemistry, the standard makes no substantive advance beyond solutions that were available on the Web in the mid 1990s, but which have recently been surpassed by the capabilities of assistive technologies. A notable qualification to this conclusion is the re- cently introduced success criterion 1.4 .10 , which is designed to facilitate reading of content by people who have low vision by ensuring that, when enlarged, it is wrapped appropriately to fit the size of the viewport. In some cases, this requirement may apply to mathematical or chemical notation (White, 2019), but the extent of its relevance is limited by the exception for "parts of the content which require twodimensional layout for usage or meaning".

As the implications of WCAG for mathematical content illustrate, the durability of the standard has its limits. Although the success criteria were designed to be independent of specific Web technologies and therefore to remain effective and relevant over time, assumptions about the capabilities of these technologies are nevertheless reflected in the requirements, sometimes subtly. In important respects, including the case of specialized notations, these assumptions have not been revised since the publication of WCAG 2.0 in 2008 (World Wide Web Consortium, 2008b), or indeed that of WCAG 1.0 in 1999 (World Wide Web Consortium, 1999). Significantly, they remain unchanged in WCAG 2.1, and in the public working draft of WCAG 2.2 (World Wide Web Consortium, 2020b). An informative contrast may be drawn with the National Instructional Materials Accessibility Standard (NIMAS) (United States, 2004), which prescribes an electronic format used in the United States for the delivery of textbooks that are accessible to students with print disabilities. On the one hand, the NIMAS standard, unlike WCAG, makes prescriptions at a much more detailed, technical level, specifying that the Z39.86 document format (American National Standards Institute and National Information standards Organization, 2005) must be used. Subsequently to the entry into operation of the NIMAS standard, however, the publishing industry, including advocates of accessibility, has moved progressively toward the EPUB publication format (World Wide Web Consortium, 2018a) as the preferred means of delivering accessible books. This development is not yet reflected in NIMAS, which may need to be updated, especially as the accessibility-related features of EPUB evolve to exceed those of earlier formats. On the other hand, NIMAS does include support for MathML, and the 2012 edition of the Structure Guidelines (DAISY Consortium, 2012) referred to by the standard specifies detailed requirements for its appropriate usage in the creation of accessible content. Thus, the extent to which the needs of STEM disciplines are reflected in different accessibility standards, and in their revision, reflects differences in the priorities of the organizations and communities responsible for their development. For STEM content to be taken adequately into account in future standards, more consistent representation by those with expertise in these domains will be needed in standards negotiations.

\section{Graphical Content}

Accessibility issues associated with graphics occupy a prominent place in the WCAG 2.1 success criteria. Images in 
general are a conspicuous instance of what is more broadly referred to in the Guidelines as "non-text content". Subject to exceptions which need not be considered in detail here, success criterion 1.1.1 specifies that non-text content must have "a text alternative that serves the equivalent purpose". A textual description of sufficient quality can meet this requirement. Descriptions of video are treated separately according to the level of conformance to be attained, and according to the nature of the video-specifically, whether it is live or prerecorded, and whether it is synchronized with an audio track. ${ }^{17} \mathrm{~A}$ notable limitation is that only at level AAA does WCAG require "extended" audio descriptions of video, in which the video is paused to accommodate spoken descriptions that cannot fit within preexisting pauses occurring in the foreground audio. In STEM fields, video requiring such extended audio descriptions is likely to occur frequently as a result of graphical content that cannot adequately be described or explained in a brief phrase or sentence. It follows that conformance of such videos at level AA, as may be required by policy, falls short of supporting effective nonvisual access.

Success criterion 1.4.1 excludes the use of color as the "only visual means" of communicating information, a requirement that applies to text and graphics alike. Subject to a significant exception for cases in which a specific presentation is "essential", a minimum contrast ratio is specified by success criterion 1.4.11 for "[p]arts of graphics required to understand the content". Together, these provisions are intended to enhance the visual presentation of graphics, benefiting in particular people who have low vision or who are color blind.

Although WCAG 2.1 thus includes a variety of provisions designed to improve the accessibility of graphical content, there are well known strategies for making images accessible that the Guidelines do not address. Tactile graphics and sonification are absent altogether. Nevertheless, these nonvisual alternatives are well accepted, particularly in STEM disciplines, and arguably confer advantages in understanding and interpreting complex graphical material that cannot be equaled by textual descriptions alone. Nor do the Guidelines encourage the use of vector-based image formats, notwithstanding their ability to retain a high quality of visual presentation when graphics are enlarged, or when presented on devices that vary greatly in resolution. Support for vector graphics formats is however widespread in Web browsers as well as in desktop and mobile applications. Sonification is likewise well supported in as much as synthesized audio can readily be generated in a wide range of contexts, including the programming environment of the browser (World Wide Web Consortium, 2020a). Embossers capable of producing tactile graphics are available commercially, although there is as yet no standard file format for

${ }^{17}$ See the different cases distinguished in success criteria 1.2.1, 1.2.3, 1.2.5, 1.2.7, and 1.2.8. representing tactile images (the available tactile graphics formats are proprietary to specific hardware vendors). In general, the necessary infrastructure is in place for implementing approaches to the accessibility of images that extend beyond the solutions recognized in the Guidelines. Appraising the extent to which WCAG supports the accessibility of graphical material arising in STEM domains is evidently more a question of what is omitted than a question of the limitations of the existing success criteria.

In addition, the focus of the Guidelines is largely on making graphical content accessible to people with vision-related disabilities. The positive potential of graphics to facilitate comprehension - especially by people with learning or cognitive disabilities - is not directly addressed. The role of graphics in effectively communicating ideas is especially evident in STEM disciplines. This raises the question of whether it is possible to develop guidance regarding how best to use graphics as vehicles for enhancing the understandability of materials by people with learning or cognitive disabilities, that would be suitable for inclusion in general technical standards. Standards such as WCAG are written to be independent of assumptions about the social context in which documents, multimedia, and interactive interfaces are used. Technical accessibility requirements are assumed to be independent of the audience for whom particular digital materials are intended. Whether the audience consists of primary school students, postgraduate researchers, or experienced professionals in STEM-related fields, the technical requirements are taken to be the same. Under this assumption, it is unclear whether general guidance on communicating graphically to facilitate understanding by people with learning or cognitive disabilities could usefully be given. Perhaps this problem should be regarded as indicative of the limitations of offering generic guidance which is abstracted from the author's choice of purpose and audience in developing digital materials. It could be argued on this basis that some aspects of future technical accessibility standards ought to be specific to particular audiences or social contexts, such as educational environments, rather than completely independent of these considerations. Whether this is so is a question that merits further research.

\section{WCAG: Concluding Observations}

WCAG provides some support for the accessibility of each of the distinctive aspects of materials in STEM disciplines. However, particularly in the case of linguistic components of scientific and technical communication, this support is only offered at level AAA, a prioritization which ensures in practice that it is likely to be ignored by most implementers. Provisions applicable to the accessibility of specialized notation, such as mathematical expressions, do not take advantage of the capabilities of current assistive technologies, such as the rendering of MathML markup in simultaneous speech and braille, structure-based reading and navigation, 
or synchronized highlighting. The only nonvisual alternatives to graphical content acknowledged by the Guidelines are textual descriptions, notwithstanding the wider variety of practically available options. Video content necessitating detailed descriptions is only properly supported at level AAA. The qualitative advantages of vector-based graphics formats could also be better taken into account. The positive role of graphical presentations in facilitating comprehension, including the understanding of materials in STEM domains, raises fundamental questions about the limitations of generic technical guidelines in enhancing accessibility, while indicating an as yet inadequately explored opportunity for further research and development of standards.

As was introduced earlier, the four principles according to which the Guidelines are organized state that Web content should be "perceivable", "operable", "understandable" and "robust". The inadequacies of WCAG that have been identified here in enabling the accessibility of STEM materials can be examined in relation to these principles. Upon doing so, it becomes apparent that many of the difficulties which have been discussed in this commentary have a distinctively cognitive nature. This observation is obviously true of the issues emerging from the linguistic properties of scientific and technical text, which fall into the "understandable" category. They are precisely questions of linguistic comprehension which, while relevant to all readers, have disproportionate implications for people with learning or cognitive disabilities. Although the combination of captions with sign language may be superior to the former alone in supporting the understanding of STEM content by people who are deaf, significant issues of linguistic understanding are likely to remain for some, but not all such individuals. The limitations that have been noted in the support offered by the Guidelines for the accessibility of specialized notations are also of a highly cognitive character. The principal advantages of having a correct braille representation of mathematical notation, of navigating a spoken representation according to its logical structure, or of having visual highlighting that is synchronized with a spoken rendering, all lie in the enhancements of understanding which these access strategies are intended to bring. Enhanced understanding and cognitive tractability are of the highest importance if, as is typically the case, the user is engaged in an intellectually challenging task, such as solving a mathematical problem or learning unfamiliar concepts. Implementing the strategies which are at present poorly supported by WCAG makes the notation no more perceivable than it otherwise would have been, but it does promise valuable cognitive benefits. Expanding the repertoire of nonvisual alternatives to images beyond textual descriptions to include techniques such as tactile graphics and sonification likewise has significant potential to facilitate the understanding of graphical content. Complex or dynamically generated graphs and charts, for instance, may be better understood in a tactile or sonified form than as a description, or as a mere table of values. In- deed, the most cognitively effective representation is likely to depend on the knowledge and skills of the user, as well as on the nature of the task to be performed. What should be appreciated, however, is the decisive centrality of cognitive issues in determining what access strategy, or combination of strategies is appropriate. These problems, which are as yet unaddressed by WCAG, genuinely lie at the intersection of sensory and cognitive concerns. They raise the question not just of how to make content nonvisually accessible, but also of how to make it cognitively manageable while doing so. The communicative potential of graphics for people with learning or cognitive disabilities remains unaddressed, due to the difficulty of devising general guidelines for the appropriate design and inclusion of graphical material.

A further strategic challenge in developing technical standards and policies that better support the cognitive dimension of accessibility emerges from questions of demarcation. To what extent should issues of learning and cognition be addressed by educational strategies intended to enhance the knowledge and skills of people with disabilities, and to what extent can and indeed should they be resolved by requiring the removal of barriers to effective comprehension in the design and construction of the digital content itself? ${ }^{18}$ Accessibility standards such as WCAG are built upon implicit assumptions about the knowledge and skills that users possess, and which enable them to benefit from the accessibilityrelated features that the standards prescribe. However the balance is reached between what should be provided by way of accessible design and development, and what challenges should be overcome by educational interventions, practical accessibility necessitates complementary contributions on both sides of this dichotomy. Accessibility-related characteristics of digital materials, including those in STEM disciplines, are only beneficial to people who possess the prerequisite skills needed for their effective use; and enabling these skills to be put in place is ultimately an educational responsibility

Currently, the World Wide web Consortium's Accessibility Guidelines Working Group is in the early stages of creating a redesigned set of Guidelines, to be known as WCAG 3.0 (World Wide Web Consortium, 2021). As this work progresses further, it can be expected to create opportunities to revisit technical assumptions inherent in earlier versions of the standard, and to satisfy more comprehensively the needs related to content in STEM disciplines. For this to occur, however, consistent engagement in the standardization process by accessibility specialists with expertise in these matters will doubtless be necessary.

\footnotetext{
${ }^{18}$ See Mason and Hedin (2011) for an overview of educational measures that have been proposed to facilitate comprehension of linguistically complex scientific material by students with learning disabilities.
} 


\section{An Alternative Approach to Standardization: Specifying Organizational Policies and Processes}

The Web Content Accessibility Guidelines prescribe technical requirements. From this perspective, accessibility is treated as at least in part a measurable aspect of a product or service that can be assessed by applying technical criteria. These criteria are independent both of the design and development process, and of the social context in which technology is used. From an alternative standpoint, however, accessibility can be regarded as emerging from an appropriate design and development strategy which is founded on an understanding of the needs of a diversity of users - including those with disabilities - and the practical purposes that a technological artifact is meant to serve. ${ }^{19}$ The accessibilityrelated characteristics of a product thus emanate from its design process, which is ultimately determined by the policies that its creators endorse. The causal connections run from policies, through the development process, to the specific technical characteristics that satisfy the access needs of users with disabilities as embodied in a product. Moreover, the design and development process can be sensitive to the particulars of the social conditions and expected backgrounds of those foreseen as potential users. An effective policy also seeks to ensure that accessibility requirements are satisfied if products and services are procured from sources external to the organization that uses or deploys them. Standardizing elements of the organizational policy and the development process has great potential to improve outcomes.

The ISO/IEC 30071-1:2019 (International Organization for Standardization and International Electrotechnical Commission, 2019) standard instantiates this approach by addressing aspects of an organization's accessibility policy, and through it, the integration of suitable conduct into every stage of design, development, and maintenance. The standard describes specific "activities" related to accessibility that should be included in whatever development process the organization follows. By way of a general summary, (International Organization for Standardization and International Electrotechnical Commission, 2019, § 8) these activities involve reaching an understanding of who the potential users of a product are, including people with disabilities, then defining the goals that users have and the tasks they can perform to achieve these goals. Users' accessibility needs are understood in relation to their goals and the tasks through which the latter are accomplished. On the basis of this understanding, the accessibility requirements for a product can be specified, as can criteria for evaluating whether the system's design and implementation enable users with different access

\footnotetext{
${ }^{19}$ The two standpoints, according to which accessibility can be regarded as emerging from an appropriate design process, and as necessitating the satisfaction of a set of technical criteria, should not be understood as mutually exclusive. Indeed, as will appear in the following discussion, they can be implemented in an integrated fashion.
}

needs to achieve their goals. Implementation and testing are closely interrelated activities based on the accessibility requirements. The accessibility-related functionality is also to be documented in a manner that is accessible to users via the product itself (e.g., through a help facility included in the user interface). After a product is made available to users, it may be further maintained and enhanced. During this process, the accessibility-related functionality should be preserved, and it should also be subject to improvement. The standard emphasizes that the development process should be set up to accomplish more than merely meeting technical accessibility requirements such as those found in WCAG. Rather, the objective is to ensure that users can achieve their goals in using the product with "effectiveness", "efficiency", and, ideally, "satisfaction". Thus, although an organization's policies and the approach taken to design and development can incorporate WCAG and other sources of technical requirements, the ISO standard focuses the development process decisively on ensuring that users" accessibility-related needs are met, and that this takes place in the practical context of using a product to achieve desired objectives.

A wide range of users" accessibility needs is documented and classified in an associated publication, ISO/IEC 291381:2018 (International Organization for Standardization and International Electrotechnical Commission, 2018). The needs are presented in general terms, leaving the task of interpreting and applying them in specific contexts to the skill of content and software developers. For example, in anticipating the accessibility needs of users of a proposed product, the generic list of needs in this document can provide important insights. Understanding the needs of users with disabilities is fundamental to designing accessible technological artifacts, especially in circumstances in which well known solutions to the challenges that arise are not available. As software supporting STEM disciplines becomes more complex and offers more diverse means of interaction, these situations can reasonably be expected to occur with increasing frequency. Virtual reality, augmented reality, interactive simulations, speech-based interfaces, and multimodal systems are among the trends in human-computer interaction that move beyond traditional graphical user interfaces, for which the problems of accessibility are relatively well understood. Even in the case of conventional graphical interfaces, achieving a level of accessibility for a reasonably wide variety of users which consistently provides for "effectiveness", "efficiency" and "satisfaction" necessarily engages designers and developers in understanding what users" needs are, and how they can best be satisfied. Although this quality of work demands more knowledge and skill of the software developer or content author than does satisfying a list of relatively well defined technical requirements as in WCAG, the opportunities to create products that more fully meet the needs of users are correspondingly greater. The ISO/IEC 30071-1:2019 standard may thus be more difficult for insufficiently knowledgeable and experienced practitioners to 
apply, but it has the advantage of focusing firmly on the ultimate aim - satisfying users' accessibility needs - and it accomplishes this in a way that can be incorporated into an organization's policies and practices. This indeed provides a pathway to pervasive accessibility, to making inclusion of people with disabilities a routine result of design and development activities. This conclusion is true just as much in connection with hardware, software and documents in STEM domains as it is in more general settings.

\section{Remarks on the Legal Underpinnings of the Ac- cessibility of STEM Materials}

The application of anti-discrimination laws and other regulatory requirement to the accessibility of hardware, software and documents developed for use in STEM disciplines is a complex matter. It is impossible to do justice to this topic within the bounds of a general paper. A comparative appraisal of the regulatory provisions operating in different countries would surely be informative in shaping the further development of policy, not only to enhance the accessibility of materials in STEM fields but in improving accessibility generally. In the absence of such a review, the discussion here shall be confined to two remarks: first, an acknowledgment of the relevant, foundational principles of international human rights law, and second, a brief example of their recent application in the European Accessibility Act, which has apparent implications for the accessibility of publications in STEM disciplines. The United Nations Convention on the Rights of Persons with Disabilities (CRPD) (United Nations, 2006) normatively recognizes the human rights of people with disabilities under international law. ${ }^{20}$ Article 9 of the CRPD sets forth a right to accessibility. States Parties are obligated, in part, "to ensure to persons with disabilities access, on an equal basis with others, to [...] information and communications, including information and communications technologies and systems". They are also required to "take appropriate measures [...] [t]o promote access for persons with disabilities to new information and communications technologies and systems, including the Internet" (paragraph $2 \mathrm{~g}$ ) and "[t]o promote the design, development, production and distribution of accessible information and communications technologies and systems at an early stage, so that these technologies and systems become accessible at minimum cost" (paragraph $2 \mathrm{~h}$ ).

As Seatzu (2017) has noted, the right to accessibility expressed in article 9 is important to the exercise of other rights recognized by the CRPD, including the rights to education (article 24) and to employment (article 27), respectively. Indeed, article 3 acknowledges "accessibility" as a general principle of the CRPD. These interrelated and mutually supporting human rights constitute a legal basis for States Parties to the CRPD to take measures that facilitate

${ }^{20}$ For details of which nation states have signed and ratified the Convention, see United Nations (n.d.). access to information and communication technology-based goods and services encountered by people with disabilities, including those which arise in education and employment. By implication, the scope of what should be made accessible to people with disabilities includes materials in STEM disciplines. The generality of the right to accessibility is reinforced in article 9 of the CRPD by its broad application, not only to information and communication technology-based systems, but also to "other facilities and services open or provided to the public". ${ }^{21}$ Of course, to have full practical effect, these human rights need to be implemented within the domestic legal system of each State Party, and realized through the carrying out of appropriate policies. A review of the extent to which the relevant human rights obligations are implemented in domestic legal systems is beyond the scope of this paper, as is discussion of the degree to which the laws of countries that have not ratified the CRPD, such as the United States, are nevertheless consistent with its principles.

A recent and prominent example of legislative efforts to advance the right to accessibility is the European Accessibility Act (European Union, 2019). The duties of the European Union under the CRPD are explicitly noted in the preamble, although the principal purpose of the Act (article 1) is to harmonize accessibility-related requirements among the European Union's Member States in support of free trade within the common market. Certain products and services offered after 28 June 2025 are subject to a series of obligations with respect to accessibility, as specified in Annex I of the Act. It is significant that, under article 2, the scope of the Act includes "e-readers" as well as "e-books and dedicated software". These provisions are sufficiently broad to include, for example, STEM-related books used in educational or professional contexts. The Act provides for harmonized European accessibility standards to be created, conformity with which is presumed to satisfy the accessibility requirements (article 15). Once these standards have been issued, it may be feasible to assess more fully the implications of the European Accessibility Act for STEM-related publications.

The prescriptions of Annex I related to e-books are relatively general. Echoing the four principles of the Web Content Accessibility Guidelines, the requirements related to electronic books (Annex I, section Iv (f)) include a provision "allowing alternative renditions of the content and its interoperability with a variety of assistive technologies, in such a way that it is perceivable, understandable, operable and robust". In connection with STEM content, this requirement is open to multiple interpretations regarding the extent of the support for accessibility that is needed. The more detailed European standards may clarify the interpretive question considerably. This legislation affects not only European publishers, but also publishers from outside the Union whose products are

\footnotetext{
${ }^{21}$ Commentary can be found in Seatzu (2017).
} 
placed on the European common market. Its consequences for accessibility are likely to be noticed both within and outside the European Union, especially having regard to the fact that it would be cost effective for publishers to offer the same product to a global market, hence delivering the same accessibility features to all users irrespective of their location.

\section{Conclusions}

Pervasive accessibility of STEM-related products and services can only be realistically achieved by putting into place a variety of factors. These factors include suitable standards and policies, education of practitioners, and further development of knowledge concerning how to make materials in STEM disciplines generally accessible to people with disabilities. STEM content has specific characteristics, such as linguistic complexity, specialized notations, and graphical conventions, which together pose unique challenges for the design and implementation of accessible solutions. Arriving at a better understanding of these challenges and of how best to resolve them in particular contexts is a proper role for technical research in accessibility. This research should be grounded in the needs and perspectives of students and professionals in STEM domains who have disabilities - individuals who are uniquely well placed to contribute to such an understanding. ${ }^{22}$

As has been argued here, existing standards, notably WCAG and policies that inherit its requirements, provide only partial support for the accessibility of STEM content relative to the state of the art. The process and policy-oriented standard, ISO/IEC 30071-1:2019, has substantial potential in the hands of skilled developers of accessible information technologies to advance the accessibility of STEM materials. Nevertheless, greater engagement in standards negotiations by specialists in the accessibility issues emerging from the needs of students and professionals with disabilities in STEM disciplines is clearly justified. Without effective advocacy, there is a risk that these issues will not receive the necessary dedication of effort as international standards continue to evolve. The development of WCAG 3.0 creates one opportunity, among others, to exercise a positive influence in negotiations.

The accessibility of STEM content is well supported by the application of foundational provisions of international disability rights law, particularly in its recognition of a general right to accessibility. Further, the inclusion of electronic books and reading technologies within the scope of the European Accessibility Act offers an opportunity for policy to enhance the accessibility of STEM-related publications.

In general, reaching a state of social and technological development in which STEM materials are pervasively accessible

\footnotetext{
${ }^{22}$ Shew (2020) capably articulates the case for strong involvement by people with disabilities in the development of technologies designed to meet their needs.
}

would involve a strategic and persistent effort by parties with different types of expertise, leading to sustained changes in policies, practices, and technologies. Collaborative engagement by a diverse community of researchers, technology developers, policy specialists, standard negotiators, educators, and people with disabilities, among others, is called for in advancing this objective. Despite the undoubted progress of recent decades, it is apparent that considerable work remains.

\section{Acknowledgments}

The author gratefully acknowledges Mark Hakkinen, Heather Buzick, Danielle Guzman-Orth, and Melissa Gholson of Educational Testing Service for reviewing the manuscript. The entire responsibility for the opinions expressed herein rests, however, with the author.

\section{References}

Ahlberg, J. (2014). Educational justice for students with cognitive disabilities. Social Philosophy \& Policy,31(1), 150 .

American National Standards Institute and National Information standards Organization. (2005).

Ansi/nisoz39.86- 2005 (r2012) - specifications for the digital talking book. Retrieved from

http://www.daisy.org/z3986/2005/Z3986-2005.html

Benetech. (2015). Born accessible and the new golden age of inclusive education. Retrieved October 28, 2020, from https://benetech.org/born-accessible-and-the-new-goldenage-of-inclusive-education/

Bragg, D., Koller, O., Bellard, M., Berke, L., Boudreault, P., Braffort, A., ... Verhoef, T., et al. (2019). Sign language recognition, generation, and translation: An interdisciplinary perspective. In The 21st international acm sigaccess conference on computers and accessibility (pp. 16-31).

Brighouse, H., Ladd, H. F., Loeb, S., \& Swift, A. (2018). Educational goods: Values, evidence, and decision-making.University of Chicago Press.

DAISY Consortium. (2012). Daisy 3 structure guidelines. Retrieved from https://www.daisy.org/z3986/ structure/SG-DAISY3/index.html

Debevc, M., Milošević, D., \& Kožuh, I. (2015). A comparison of comprehension processes in sign language interpreter videos with or without captions. PloS one, 10(5), e0127577.

Ellcessor, E. (2015). Blurred lines: Accessibility, disability, and definitional limitations. First Monday.

European Telecommunications Standards Institute. (2018). En 301549 v2.1.2.

European Union. (2016). Directive (eu) 2016/2102 of the 


\section{Journal of Sclence Education}

european parliament and of the council of 26 october 2016 on the accessibility of the websites and mobile applications of public sector bodies. Official Journal of the European Union, L 327, 1-15.

European Union. (2019). Directive (eu) 2019/882 of the european parliament and of the council of 17 april 2019 on the accessibility requirements for products and services. Official Journal of the European Union, L 151, 70.18

Fajardo Flores, S., \& Archambault, D. (2012).

Understanding algebraic manipulation: Analysis of the actions of sighted and non-sighted students. In The international workshop on digitization and e-inclusion in mathematics and science (Vol. 2012). Nihon University Tokyo, Japan.

Hansen, E. G., Zapata-Rivera, D., \& White, J. (2018). Framework for the design of accessible intelligent tutoring systems. In S. D. Craig (Ed.), Tutoring and intelligent tutoring systems (pp. 69-100). Nova Science Publishers.

International Organization for Standardization and International Electrotechnical Commission. (2018). Iso/iec 29138-1:2018 information technology - user interface accessibility - part 1: User accessibility needs.

International Organization for Standardization and International Electrotechnical Commission. (2019). Iso/iec 30071-1:2019 information technology - development of user interface accessibility - part 1: Code of practice for creating accessible ict products and services.

Lewis, P., Noble, S., \& Soiffer, N. (2010). Using accessible math textbooks with students who have learning disabilities. In Proceedings of the 12th international acm sigaccess conference on computers andaccessibility (pp. 139-146). ACM.

Lewthwaite, S., \& Sloan, D. (2016). Exploring pedagogical culture for accessibility education in computing science. In Proceedings of the 13th web for all conference (pp. 1-4).

Liasidou, A. (2013). Intersectional understandings of disability and implications for a social justice reform agenda in education policy and practice. Disability 83 Society, 28(3), 299-312.

Marschark, M., Sapere, P., Convertino, C. M., Mayer, C., Wauters, L., \& Sarchet, T. (2009). Are deaf students'reading challenges really about reading? American annals of the deaf, 154(4), 357-370.

Mason, L. H., \& Hedin, L. R. (2011). Reading science text: Challenges for students with learning disabilities and considerations for teachers. Learning Disabilities Research Es Practice, 26(4), 214-222.

Meyer, K. (Ed.). (2014). Education, justice and the human good: Fairness and equality in the education system.

Routledge.Ong-Dean, C. (2009). Distinguishing disability:
Parents, privilege, and special education. University of Chicago Press.

Orbit Research. (n.d.). Graphiti@ — a breakthrough in non-visual access to all forms of graphical information.Retrieved October 27, 2020, from https://www.orbitresearch.com/product/graphiti/

Persson, H., Åhman, H., Yngling, A. A., \& Gulliksen, J. (2015). Universal design, inclusive design, accessible design, design for all: Different concepts - one goal? on the concept of accessibility - historical, methodological and philosophical aspects. Universal Access in the Information Society, 14(4), 505-526.

Schneiderwind, J., \& Johnson, J. M. (2020). Disability and invisibility in stem education. Journal of Higher Education Theory $\&$ Practice, 20(14).

Seatzu, F. (2017). Article 9 [accessibility]. In V. D. Fina, R. Cera, \& G. Palmisano (Eds.), The united nations convention on the rights of persons with disabilities (pp. 225-242). Springer.

Shew, A. (2020). Ableism, technoableism, and future ai. IEEE Technology and Society Magazine, 39(1), 40-85.

Silliman, E., \& Wilkinson, L. C. (2015). Challenges of the academic language register for students with language learning disabilities. In R. H. Bahr \& E. R. Silliman (Eds.), Routledge handbook of communication disorders (p. 291). Taylor and Francis.

Smith, T. L., Greenberg, J., Reid, S., \& Moore, E. B. (2018). Parallel dom architecture for accessible interactive simulations. In Proceedings of the internet of accessible things (pp. 1-8).

Soiffer, N., \& Noble, S. (2019). Mathematics and statistics. In S. Harper \& Y. Yesilada (Eds.), Web accessibility - a foundation for research (pp. 417-443). Springer.

Stöger, B., Batusic, M., Miesenberger, K., \& Haindl, P. (2006). Supporting blind students in navigation and manipulation of mathematical expressions: Basic requirements and strategies. In International conference on computers for handicapped persons (pp. 1235-1242). Springer.19

Stöger, B., Miesenberger, K., \& Batušić, M. (2004). Mathematical working environment for the blind motivation and basic ideas. In International conference on computers for handicapped persons (pp. 656-663).Springer.

Taylor, A. (2018). The logic of deferral: Educational aims and intellectual disability. Studies in Philosophy and Education, 37 (3), 265-285.

United Nations. (n.d.).Untc-depositary. Retrieved November 2, 2020, from

https://treaties.un.org/Pages/ViewDetails.aspx?src= TREATY\&mtdsgno $=\mathrm{IV}-15 \&$ chapter $=4 \&$ clang $=\mathrm{en}$ 


\section{Journal of Sclence Education}

United Nations. (2006). Convention on the rights of persons with disabilities. United Nations treaty Series,2515, 3 .

United States. (2004). $34 \mathrm{cfr}$ appendix c to part 300 - national instructional materials accessibility standard(nimas).

United States. (2017). 36 cfr part 1194-information and communication technology standards and guidelines.

Völkel, T., Weber, G., \& Baumann, U. (2008). Tactile graphics revised: The novel brailledis 9000 pin-matrix device with multitouch input. In International conference on computers for handicapped persons (pp. 835-842). Springer.

Wentz, B., Jaeger, P. T., \& Lazar, J. (2011). Retrofitting accessibility: The legal inequality of after-the-fact online access for persons with disabilities in the united states. First Monday.

White, J. J. (2019). Wcag 2.1 meets stem: Application, interpretation, and opportunities for further standard development. Journal of Science Education for Students with Disabilities, 22(1), n1.

White, J. J. (2020). The accessibility of mathematical notation on the web and beyond. Journal of

ScienceEducation for Students with Disabilities, 23(1), n1.

World Wide Web Consortium. (1999). Web content accessibility guidelines 1.0. Retrieved from https://www.w3.org/TR/WCAG10/

World Wide Web Consortium. (2008a). Pronunciation lexicon specification (pls) version 1.0. Retrieved April 20, 2020, from

https://www.w3.org/TR/pronunciation-lexicon/

World Wide Web Consortium. (2008b). Web content accessibility guidelines (wcag) 2.0. Retrieved from https://www.google.com/patents

World Wide Web Consortium. (2014). Mathematical markup language (mathml) version 3.0 2nd edition. Retrieved from

http://www.w3.org/TR/2014/REC-MathML3-20140410/

World Wide Web Consortium. (2015). Authoring tool accessibility guidelines (atag) 2.0. Retrieved from https://www.w3.org/TR/ATAG20/

World Wide Web Consortium. (2017). Accessible rich internet applications (wai-aria) 1.1. Retrieved from https://www.w3.org/TR/wai-aria-1.1/

World Wide Web Consortium. (2018a). Epub 3.2. Retrieved April 20, 2020, from https://www.w3.org/ publishing/epub3/epub-spec.html

World Wide Web Consortium. (2018b). Web content accessibility guidelines (wcag) 2.1. Retrieved from https://www.w3.org/TR/2018/REC-WCAG21-20180605/
World Wide Web Consortium. (2020a). Web audio api. Retrieved from https://www.w3.org/TR/2020/CRwebaudio-20200611/

World Wide Web Consortium. (2020b). Web content accessibility guidelines (wcag) 2.2. Retrieved from https://www.w3.org/TR/2020/WD-WCAG22-20200811/

World Wide Web Consortium. (2021). W3c accessibility guidelines (wcag) 3.0. Retrieved from https://www.w3.org/TR/2021/WD-wcag-3.0-20210121/

Yoon, J.-O., \& Kim, M. (2011). The effects of captions on deaf students' content comprehension, cognitive load, and motivation in online learning. American annals of the deaf, 156(3), 283-289. 